\title{
Toward UAV-based communication: improving throughput by optimum trajectory and power allocation
}

\author{
Sedighe Nasrollahi and Seyed Masoud Mirrezaei* (i)
}

\author{
*Correspondence: \\ sm.mirrezaei@shahroodut.ac.ir \\ Faculty of Electrical \\ Engineering, Shahrood \\ University of Technology, \\ Shahrood, Iran
}

\begin{abstract}
It is predicted that the use of unmanned aerial vehicles (UAVs) in communication systems will be more extensive in future generations of wireless telecommunication networks, due to their facilitating advantages. In this paper, a UAV-based wireless communication system is considered in which a UAV is employed as a relay to connect two ground users. These two disconnected users make a communication pair. Our aim is to maximize the minimum achievable information rate for the communication link between the transmitter and receiver, by cooperatively optimizing UAV trajectory and transmitter and source power allocation. Motivated by the above, we formulate the optimization problem. The solving process is complicated because of the non-convexity of the formulated problem. To overcome this difficulty, we convert the main problem to some sub-problems by fixing some constraints and solving them with iterative algorithms such as successive convex optimization and reaching the solution for the main problem. Simulation results show the capability of the proposed algorithm.
\end{abstract}

Keywords: UAVs, Relay, Optimization, Power allocation, UAV trajectory, Communication

\section{Introduction}

By increasing mobile users in 5G and also the expansion of Internet of Things (IoT) applications in future communication systems, communication networks face a high amount of data traffic that is out of available terrestrial base station's capacity. To overcome this challenge and coverage improvement of communication networks, relaying is an effective technique $[1,2]$. As most of the communication systems are wired, conventional relaying systems are based on static relaying. But it is costly to set up a terrestrial base station wherever there is a demand. So due to cost reduction, device miniaturization, and other advantages, unmanned aerial vehicles can be employed as demand-based relays in wireless networks. In addition to relaying, UAVs can be used in other applications such as power transfer[3], data collection[4], information broadcasting, cargo delivery, traffic monitoring, and emergency situations for example disaster management. In all these applications, there are two types of problem definition approaches. In one branch, the goal is to find the optimal hovering points for static UAVs. In other words, author(s) and the source, provide a link to the Creative Commons licence, and indicate if changes were made. The images or other third party material in this article are included in the article's Creative Commons licence, unless indicated otherwise in a credit line to the material. If material is not included in the article's Creative Commons licence and your intended use is not permitted by statutory regulation or exceeds the permitted use, you will need to obtain permission directly from the copyright holder. To view a copy of this licence, visit http:// creativecommons.org/licenses/by/4.0/. 
only node placement is in question $[5,6]$. On the other hand, it is desired that the maximum mobility of the drone is used to design the optimal trajectory for it. In addition to mobility, UAV-based communication systems benefit from on-demand deployment and also the line-of-sight channel between UAV and considered ground transmitter or receiver[7]. According to[8] and 3GPP [9], for the moderate altitude of UAV for example more than 50 meters, the LOS probability is more than 90 percent. So, the channel may be less affected by shadowing, fading, and multipath propagation. Since the horizontal placement of the UAV can be changed, the channel between the UAV and ground nodes has variation. Motivated by this, UAV can get closer to the objective area and achieve a better communication channel with more gain. As discussed in [10, 11], another parameter of a UAV's placement is its altitude that influences the coverage probability and system sum rate. Based on practical applications, various research endeavors have been dedicated to UAV-aided wireless networks in the last decade. In most of them, UAV is considered as a mobile base station or mobile relay to serve some ground nodes in a geographical area. In $[7,12]$, the deployment of UAV as a flying base station for a desired geographical area with both downlink users and device-to-device (D2D) users is analyzed, and the optimal height of the UAV is obtained by maximizing the coverage probability. In [13], an efficient resource allocation and trajectory optimization in NOMA wireless network are studied. A heuristic algorithm based on matching and swapping theory is used for user and UAV time scheduling, and the optimized power and trajectory are derived by solving a non-convex optimization problem. Considering UAV as a mobile relay as an extensive issue of researches mentioned in [14, 15], the authors in [16] investigated a communication network with a UAV as a relay and multiple ground pairs as the source and destination aiming to minimize the total energy consumption by jointly optimizing time and power allocation and UAV trajectory. So energy minimization is an important factor in designing UAV-enabled systems. Though initial attempts for designing energy-efficient UAV communication was appropriated to fixed-wing UAV-enabled communication system by maximizing its energy efficiency in bits/Joule [17], authors in [18] investigated energy-efficient communication design for rotary-wing UAVs in a multi-user situation. In [19-21], the scenario of multiple UAVs deployed as areal base stations and relays, respectively, was studied. Another influential role of UAV relaying is in crisis management. In natural disasters, IoT coverage will be extremely affected, due to the destruction of communications infrastructure. In such cases, having an emergency communication network can be a crucial factor in getting rid of the status quo. UAVs can be applied to implement public safety scenarios to support disaster alleviation measures [22-24]. Since UAV relaying face the challenge of malicious attacks including eavesdropping, the security issue of UAV networks is critical to have a protected communication. The authors in [25] considered boosting physical layer security by using a mobile relaying system, in which a UAV acts as a mobile relay and flexibly regulates its location to enhance the desired wireless communication security. In [2628], an optimization problem has been formulated to maximize the minimum average secrecy rate over all receivers by jointly optimizing UAV trajectory and transmit power. Lu et al. [29] uses UAV for reducing data traffic pressure by carrying mobile edge computing (MEC) servers on UAVs. A secure communication design is proposed the DualUAV-MEC system. 
The major contributions of this paper are presented as follows:

- This paper introduces a UAV-relayed communication system supporting one disconnected communication pair and finds the maximum achievable transmission rate by cooperatively optimizing UAV trajectory and source and relay transmission power. So a non-convex optimization problem is created.

- To make the problem tractable, we partition the main problem into two sub-problems and solve them by successive convex optimization techniques. Then, an overall algorithm is produced to solve two sub-problems alternately.

- The advantage of our method is that sub-problems have fewer constraints than the main problem. So the complexity and convergence speed is more.

- We obtained the optimized trajectory and transmission power and observed that power is inversely related to link distance between two nodes.

- Furthermore, we compared the results of our proposed algorithm with fixed power and trajectory manner and saw more rate is gained in the proposed method.

The rest of this paper is organized as follows. In Sect. 2, our desired UAV-relayed system model and the problem formulation are introduced. Section 3 proposes the iterative algorithm based on SCO. In Sect. 4, we present the simulation results to verify the effectiveness of the proposed algorithm. Finally, we conclude this paper in Sect. 5.

\section{System model and problem formulation}

Consider the scenario that in a disaster (or any reason that destroys the communication infrastructure) area, two disconnected ground nodes that are at a distance of $\mathrm{L}$ meters apart cannot communicate with each other due to long distance or severe blockage. Thus, a UAV relay is deployed to establish a communication link between source and destination defined by $A$ and $B$, respectively. We assume that $A$ and $B$ are located on the ground with known fixed locations and the flight height of the UAV from the ground is constant and equal to $H$ in a period of $T \mathrm{~s}$. The horizontal coordinates of $A$ and $B$ and UAV are $(0,0,0),(L, 0,0)$ and $(x(t), y(t), H)$, respectively. The analytical model of such a system is shown in Fig. 1.

Note that we assume this scenario for real-time applications such as building interconnection in emergency situations; therefore, the amplify-and-forward (AF) strategy is more suitable than decode-and-forward (DF) due to less complexity. For ease of analysis, the time horizon $T$ is discretized into $N$ equal time slots. The parameter $N$ should be chosen large enough or in other words the elemental slot length be small enough, so that the position of UAV is approximately constant at any time slot. Thus, the trajectory of UAV over $T$ can be rewritten as $(x[n], y[n]), \forall n=1, \ldots, N$. But increasing $N$ will bring more computational complexity. In fact, while choosing the value of $N$, we should consider a tradeoff between the accuracy and complexity [19]. Motivated by AF protocol, the UAV transmits data to $B$, as soon as received it from $A$. We partition each time slot into two hops. Sending data from source to the UAV happens in the first hop. Before formulating the problem, for easy access to the symbols used in the article, we first introduce our notation in Table 1. 


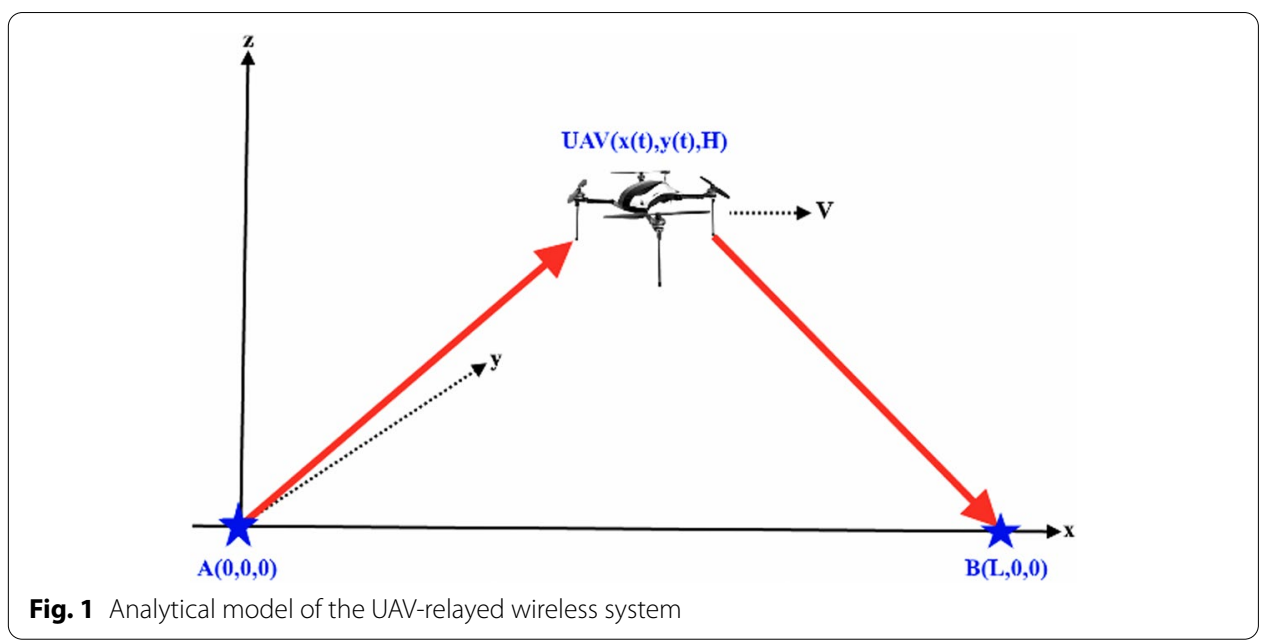

Table 1 Definitions of a subset of commonly used symbols

\begin{tabular}{ll}
\hline Symbol & Meaning \\
\hline$T$ & Flying time \\
$N$ & Number of time slots \\
$V$ & Maximum speed of UAV \\
$P_{A}[n]$ & Transmission power of $A$ \\
$X[n]$ & The transmitted signal to UAV \\
$Z_{1}[n]$ & The noise observed at UAV \\
$h_{A U}[n]$ & Channel between $\cup A V$ and $A$ \\
$d_{A U}[n]$ & Distance between $A$ and $\cup A V$ \\
$\alpha_{0}$ & Reference channel power at the distance $d_{0}=1 \mathrm{~m}$ \\
$G[n]$ & Scaling parameter \\
$P_{U}[n]$ & Transmission power of UAV \\
$h_{U B}[n]$ & Channel between UAV and $B$ \\
$Z_{2}[n]$ & The noise observed at $B$ \\
$d_{U B}$ & Distance between UAV and $B$ \\
$\overline{P_{A}}$ & Average maximum transmission power of $A$ \\
$\overline{P_{U}}$ & Average maximum transmission power of UAV \\
$\sigma^{2}$ & Power of the noise \\
\hline
\end{tabular}

The received signal at UAV in the nth time slot can be expressed as:

$$
Y_{U}[n]=\sqrt{P_{A}[n] h_{A U}[n]} X[n]+Z_{1}[n]
$$

where $P_{A}[n]$ is the transmission power of $A . X[n]$ is the transmitted signal to UAV and $Z_{1}[n] \sim N\left(0, \sigma^{2}\right)$ is the power of additive white Gaussian noise (AWGN) observed at UAV. $h_{A U}[n]$ represents the LOS channel between UAV and source. Considering the free-space pass loss model and ignoring the Doppler effect caused by UAV mobility, the channel power gain from source to UAV can be expressed as: 


$$
h_{A U}[n]=\alpha_{0} d_{A U}^{-2}[n]=\frac{\alpha_{0}}{x^{2}[n]+y^{2}[n]+H^{2}}
$$

where $\alpha_{0}$ illustrates the reference channel power at the distance $d_{0}=1 \mathrm{~m}$. As we see, the channel power depends only on the UAV-user distance. For the second hop, the UAV scales the received signal and broadcasts it to the destination with gain $G[n]$ as follows:

$$
G[n]=\sqrt{\frac{P_{U}[n]}{P_{A}[n] h_{A U}[n]+\sigma^{2}}}
$$

where $P_{U}[n]$ is the transmission power of UAV. Thus, the signal received at $B$ can be written as follows:

$$
Y_{B}[n]=G[n] \sqrt{h_{U B}[n]} Y_{U}[n]+Z_{2}[n]
$$

where $Z_{2}[n] \sim N\left(0, \sigma^{2}\right)$ is the power of additive white Gaussian noise at destination. The following equation shows the channel gain of UAV-B link:

$$
h_{U B}[n]=\alpha_{0} d_{U B}^{-2}[n]=\frac{\alpha_{0}}{(x[n]-L)^{2}+y^{2}[n]+H^{2}}
$$

In the above expressions, $d_{A U}[n]$ and $d_{U B}[n]$ are the link distance between source and UAV, and UAV and destination at time slot $n$. Considering (3) and (4), the corresponding signal-to-noise ratio (SNR) at the destination can be written as:

$$
\gamma[n]=\frac{P_{A}[n] P_{U}[n] h_{A U}[n] h_{U B}[n]}{\left(P_{A}[n] h_{A U}[n]+P_{U}[n] h_{U B}[n]+\sigma^{2}\right) \sigma^{2}}
$$

The accessible information rate for the source to destination link at nth time slot can be expressed as:

$$
R[n]=\frac{1}{2} \log _{2}(1+\gamma[n]), n=1, \ldots, N
$$

The goal is maximizing the minimum of this rate by optimizing both source/UAV power allocation and UAV trajectory. By defining $P \triangleq\left(P_{A}[n], P_{U}[n]\right)$ and $W \triangleq(x[n], y[n])$, the optimization problem can be formulated as:

$$
\begin{aligned}
& \text { (P1) : } \max _{P, W} \min R[n], n=1, \ldots, N . \\
& \text { s.t. } \sum_{n=1}^{N} P_{A}[n] \leq N \overline{P_{A}}, \sum_{n=1}^{N} P_{U}[n] \leq N \overline{P_{U}} \\
& P_{A}[n] \geq 0, P_{U}[n] \geq 0, n=1, \ldots, N . \\
& (x[n+1]-x[n])^{2}+(y[n+1]-y[n])^{2} \leq\left(\frac{V T}{N}\right)^{2}, n=1,2, \ldots, N-1
\end{aligned}
$$


where $\overline{P_{A}}$ and $\overline{P_{U}}$ are the average maximum transmission power of source A and UAV. By defining $\mathrm{V}$ as the maximum permitted flying speed of $\mathrm{UAV}, \frac{V T}{N}$ represents the maximum horizontal distance the UAV can fly in each time slot. Constraint (8d) implies that the distance that the UAV travel in one time slot should not exceed its maximum value. The max-min optimization problem is non-convex because the logarithmic objective function is not convex. In our proposed method, we suggest an iterative algorithm to solve this problem.

\section{Proposed method}

As we say, the main optimization problem is non-convex. To be more precise, the Hessian matrix of objective function has a negative value relative to the optimization variables including power and trajectory. This makes the problem intractable to solve. In this part, we introduce two sub-problems and develop an iterative algorithm to solve them alternately to achieve the solution for the main problem. In fact, separating the main problem into two sub-problems facilitates the solving process by reducing the optimization variables and their related constraints as a result. First, we solve the optimization problem with fixed UAV trajectory and obtain the source/relay power allocation and then repeat with fixed power allocation to obtain the optimal trajectory. Finally, the overall algorithm is proposed.

\subsection{Power optimization with fixed UAV trajectory}

By assuming the UAV trajectory fixed, the constraints reduce to ones that are only on the power. Also, the varying channel is known due to the pre-determined trajectory. So, the main problem can be written as the following form:

$$
\begin{aligned}
& (P 1.1): \max _{P} \min R[n], n=1, \ldots, N \\
& \text { s.t. }(11 b) \text { and }(11 c)
\end{aligned}
$$

The objective function does not change. So, the problem (P1.1) is still non-convex. To cope with this non-convexity, we utilize iterative approximation helping from successive convex optimization techniques. As mentioned in [30], any convex function is lower-bounded by its first-order Taylor expansion. Motivated by this, we maximize the lower bound of our objective function by optimizing the source and UAV's power in each iteration. By converting $\gamma[n]$ to $\frac{1}{\gamma[n]}$, the convexity is done firstly, and then we can use Taylor approximation. We can write the Taylor expansion of the transmission rate at $\frac{1}{\gamma[n]}$ as follows:

$$
\begin{aligned}
& f(x) \geq f\left(x_{0}\right)+f\left(x_{0}\right)\left(x-x_{0}\right), \forall x \rightarrow \\
& R_{l+1}[n] \geq R_{l}[n]-\frac{\gamma_{l}^{2} \log _{2} e}{2\left(\gamma_{l}[n]+1\right)}\left(\frac{1}{\gamma_{l+1}[n]}-\frac{1}{\gamma_{l}[n]}\right)=R_{l b, l+1}, n=1, \ldots, N
\end{aligned}
$$

In the above expressions, $l$ and $l+1$ indexes introduce $l$ th and $(l+1)$ th iterations. From the equation of SNR calculation, it is obvious that $\gamma[n]$ is not convex with respect to $P_{A}[n]$ and $P_{U}[n]$. So, we can say that $\frac{1}{\gamma[n]}$ is a convex function of $P_{A}[n]$ and $P_{U}[n]$. Therefore, we can rewrite the above problem as:

$$
(P 1.2): \max _{P} \min R_{l b, l+1}[n], n=1, \ldots, N .
$$




$$
\begin{aligned}
& \text { s.t. } \sum_{n=1}^{N} P_{A, l+1}[n] \leq N \overline{P_{A}} \quad, \sum_{n=1}^{N} P_{U, l+1}[n] \leq N \overline{P_{U}} \\
& P_{A, l+1}[n] \geq 0, \quad P_{U, l+1}[n] \geq 0, n=1, \ldots, N .
\end{aligned}
$$

(P1.2) is a convex version of (P1.1) and can be efficiently solved by existing standard convex optimization tools such as YALMIP. The optimal solution of (P1.1) is also lowerbounded by the solution of (P1.2).

\subsection{Trajectory optimization with fixed power}

In this part, the trajectory optimization problem is solved for any desired source or UAV power. This problem can be summarized as:

$$
(P 1.3): \max _{W} \min R[n], n=1, \ldots, N
$$

Again, we face a non-convex optimization problem because of the non-convex objective function and should utilize successive convex optimization method to find its optimal solution efficiently. We define $\left\{x_{l}[n], y_{l}[n]\right\}$ and $\left\{x_{l+1}[n], y_{l+1}[n]\right\}$, the final location of UAV after $l$ th and $(l+1)$ th iteration. Since the non-convexity of the objective function is concerning $\{x[n], y[n]\}$, we present two new variables named $s_{1}[n]=\frac{1}{h_{A U}[n]}$ and $s_{2}[n]=\frac{1}{h_{U B}[n]} \cdot \gamma[n]$ is convex with respect to $s_{1}[n]$ and $s_{2}[n]$, and first-order Taylor expansion can be used to approximate it. Firstly, we rewrite the $\gamma[n]$ according to new variables as follows:

$$
\gamma[n]=\frac{P_{A}[n] P_{U}[n]}{\left(P_{A}[n] s_{2}[n]+P_{U}[n] s_{1}[n]+\sigma^{2} s_{1}[n] s_{2}[n]\right) \sigma^{2}}
$$

Using Taylor expansion, we have

$$
\gamma_{l+1}[n] \geq \gamma_{l}[n]-C_{l}[n]\left(s_{1, l+1}[n]-s_{1, l}[n]\right)-D_{l}[n]\left(s_{2, l+1}[n]-s_{2, l}[n]\right)=\gamma_{l b, l+1}[n]
$$

where $C_{l}[n]$ and $D_{l}[n]$ are the gradients of the $\gamma[n]$ at new variables which can be calculated by

$$
\begin{aligned}
& C_{l}[n]=\frac{P_{A}[n] P_{U}[n]\left(P_{U}[n] \sigma^{2}+\sigma^{4} s_{2, l}[n]\right)}{\left(P_{A}[n] s_{2}[n]+P_{U}[n] s_{1}[n]+\sigma^{2} s_{1}[n] s_{2}[n]\right)^{2} \sigma^{4}} \\
& D_{l}[n]=\frac{P_{A}[n] P_{U}[n]\left(P_{A}[n] \sigma^{2}+\sigma^{4} s_{1, l}[n]\right)}{\left(P_{A}[n] s_{2}[n]+P_{U}[n] s_{1}[n]+\sigma^{2} s_{1}[n] s_{2}[n]\right)^{2} \sigma^{4}}
\end{aligned}
$$

From $\gamma_{l b}[n]$, we have the lower bound of transmission rate as

$$
R_{l b, l+1}[n]=\frac{1}{2} \log _{2}\left(1+\gamma_{l b, l+1}[n]\right)
$$


Now, the convex optimization problem for trajectory optimization for given source and UAV power allocation scenario can be summarized as:

$$
\begin{aligned}
& (P 1.4): \max _{W} \min R_{l b, l+1}[n], n=1, \ldots, N \\
& \text { s.t. }\left(x_{l+1}[n+1]-x_{l+1}[n]\right)^{2}+y_{l+1}[n+1]-y_{l+1}[n]^{2} \leq\left(\frac{V T}{N}\right)^{2}, n=1,2, \ldots, N-1
\end{aligned}
$$

The overall algorithm which contains the solving process of two sub-problems can be given by below algorithm:

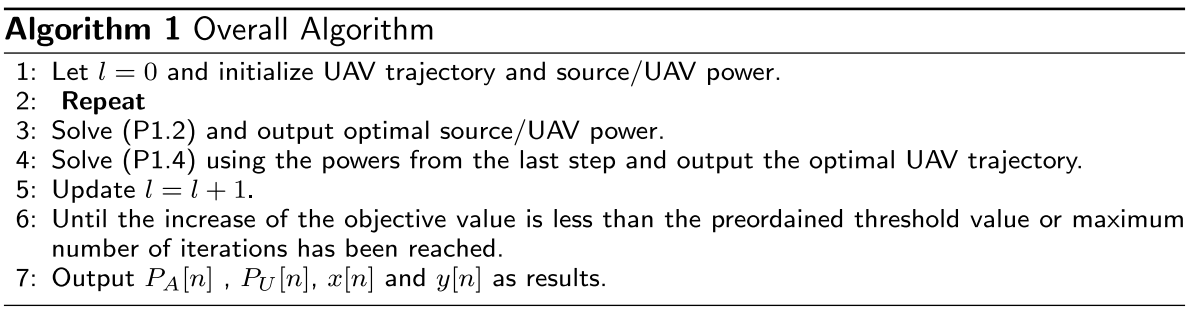

Using the above algorithm, we could solve a non-convex problem by solving two sub-convex problems in several iterations. The process is further clarified in the flowchart below:

As the flowchart shows, in the first step of the optimization problem, all the optimization variables are initialized. Then, the first sub-problem is solved with a fixed trajectory and outputs the optimal power allocation. This power is considered as a fixed power for trajectory optimization problems and after solving the second sub-problem, the optimal trajectory is obtained. Note that YALMIP solves each sub-problem with several interior iterations until results the optimal answer. After these steps, the convergence condition should be checked. We define a convergence threshold $\epsilon=10^{-6}$. If the difference of objective function value in two consecutive iterations is lower than $\epsilon$ or maximum iteration number reached, the loop will be ended and the optimal power allocation and trajectory is gained. Otherwise, the iteration number is updated, and the algorithm will be repeated until fulfilling the convergence conditions. To guarantee the convergence of the proposed algorithm, Theorem 1 is presented.

Theorem 1 The overall algorithm is guaranteed to converge, and the result is a lower bound of maximum information rate.

\section{Proof}

The results of problems (P1.2) and (P1.4) are named $K_{1}$ and $K_{2}$, respectively. As we said by using Taylor expansion and $l$ as iteration symbol, it can be verified that $K_{1, l+1} \geq K_{1, l}$ and $K_{2, l+1} \geq K_{2, l}$. The optimal values of (P1.2) and (P1.4) are nondecreasing over iteration l. If we consider $K_{3}$ as the result of the overall algorithm, we can conclude that $K_{3, l+1} \geq K_{3, l}$ at iteration $l$. This is because the result of the overall algorithm is obtained by employing $K_{1}$ and $K_{2}$ alternately. $K_{3}$ in the overall algorithm is upper-bounded by the optimal solution of (P1). So the convergence of the proposed algorithm is guaranteed. 


\section{Simulation results}

This section provides simulation results to verify the performance of the proposed algorithm. We assume the scenario that the distance between transmitter and receiver is $L=2000 \mathrm{~m}$. The altitude of the UAV is constant and equal to 100 meters. The bandwidth of the communication channel between source and destination is $20 \mathrm{MHz}$. The noise power spectral density is $-100 \mathrm{dBm} / \mathrm{Hz}$ and the value of $\alpha_{0}$ is assumed $30 \mathrm{~dB}$. The maximum speed of UAV is $60 \mathrm{~m} / \mathrm{s}$. Another assumption is that the UAV flies from $(0,0$, $100)$ to $(2000,0,100)$ in $100 \mathrm{~s}$. The maximum average transmission power at source and $\mathrm{UAV}$ are the same and equal to $10 \mathrm{dBm}$.

For the first scenario with a fixed trajectory, we assume directional trajectory from $(0$, $0,100)$ to $(2000,0,100)$ with a constant speed of $20 \mathrm{~m} / \mathrm{s}$. Figure 2 is the output of power allocation with fixed trajectory. It presents that when the UAV travels close to the source, it should transmit data with much more power because the link distance to the destination is more. In this case, the transmit power of A is less. In other words, the transmit power of A increases as the transmit power of the UAV decreases while traveling from

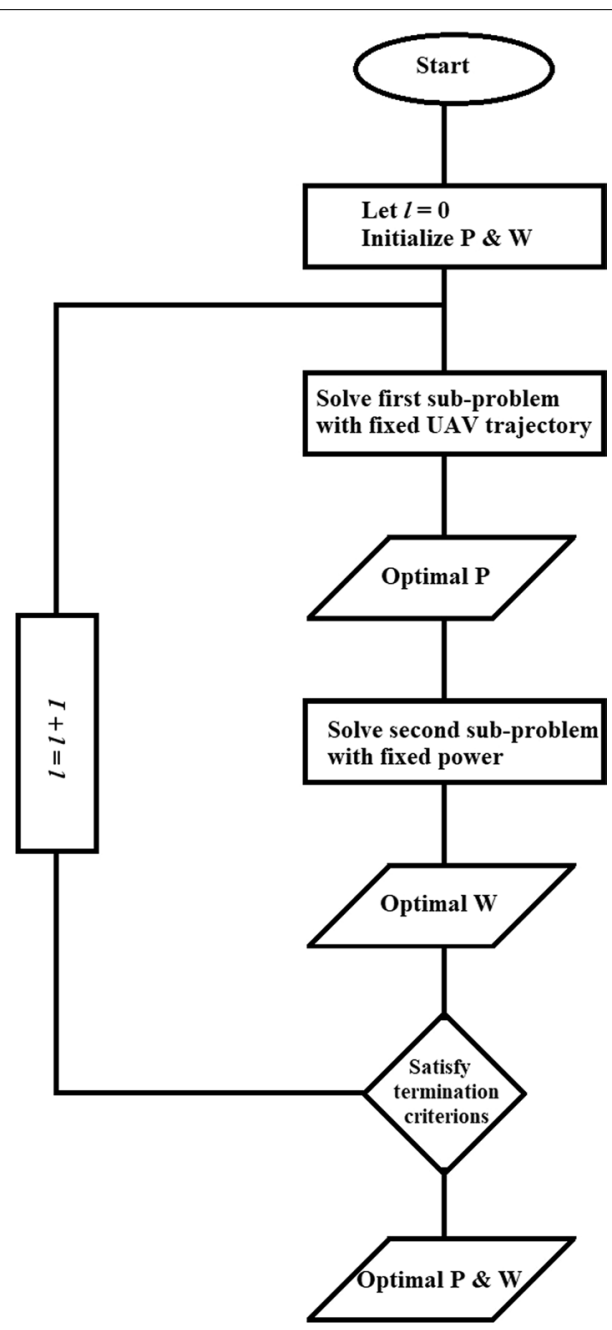

Fig. 2 Flowchart of the proposed algorithm 


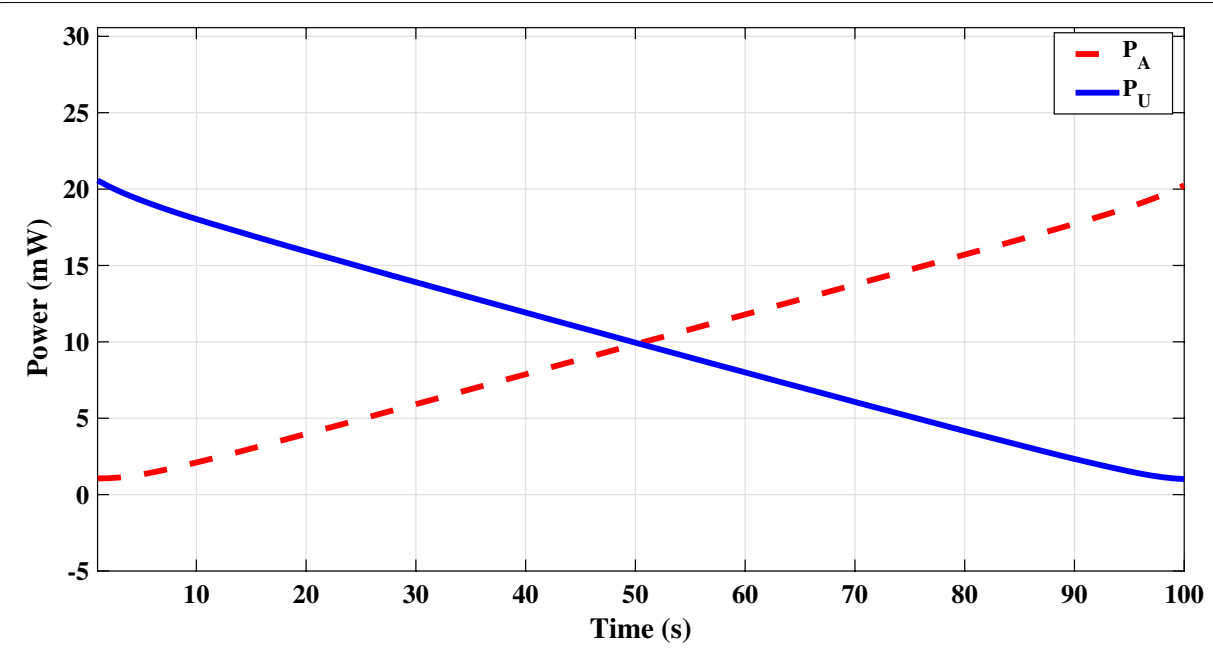

Fig. 3 Power allocation with fixed trajectory

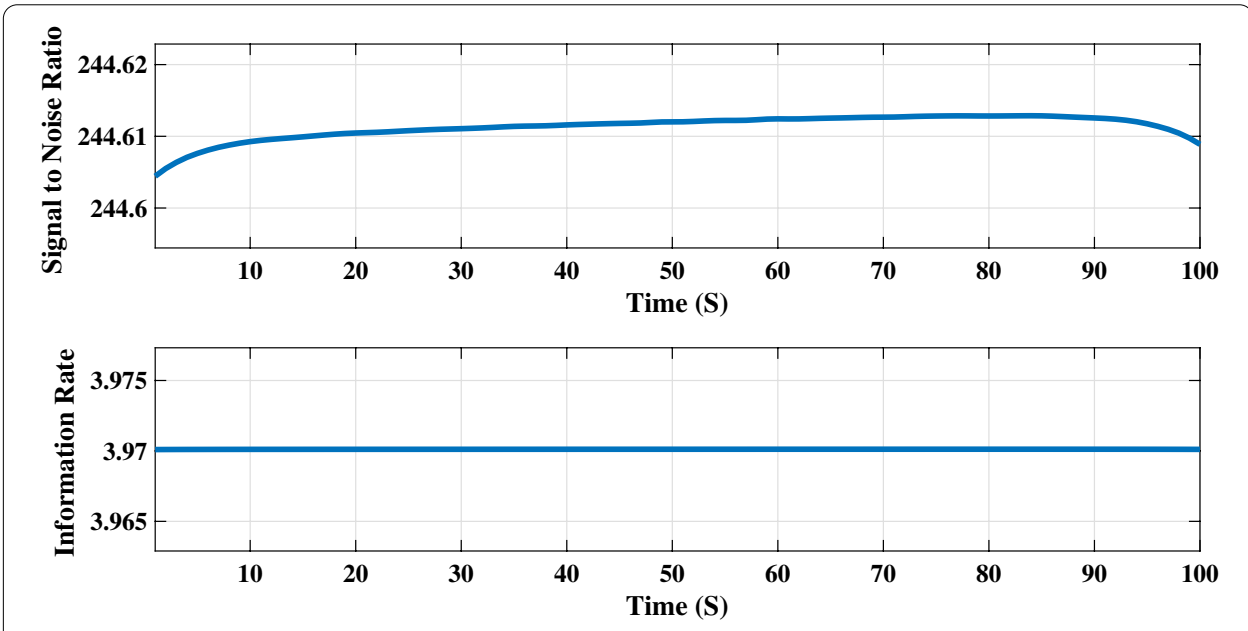

Fig. 4 Optimized SNR and information rate for power allocation with fixed trajectory

source to destination. As shown in Fig. 3, in the middle of the trajectory at time $50 \mathrm{~s}$, the power of source and UAV is equal to $10 \mathrm{~mW}$ because of equal link distance.

Figure 4 shows the achievable signal-to-noise ratio and its equivalent information rate for the optimized power with fixed trajectory situation. The optimized information rate is about $3.97 \mathrm{bits} / \mathrm{s} / \mathrm{Hz}$.

In Fig. 5, we show the value of our objective function according to iteration numbers to verify that the maximization procedure of the objective value is satisfied. As it is shown, the value of objective function goes from 3.927 to 3.97 which is the optimized information rate.

In the second phase, we check out the case that the power of A and destination are fixed and equal to $\bar{P}=10 \mathrm{~mW}$ in the whole time of flying. Figure 6 shows the optimized $x$-axis of the trajectory in ten iterations. The UAV flies with its maximum 


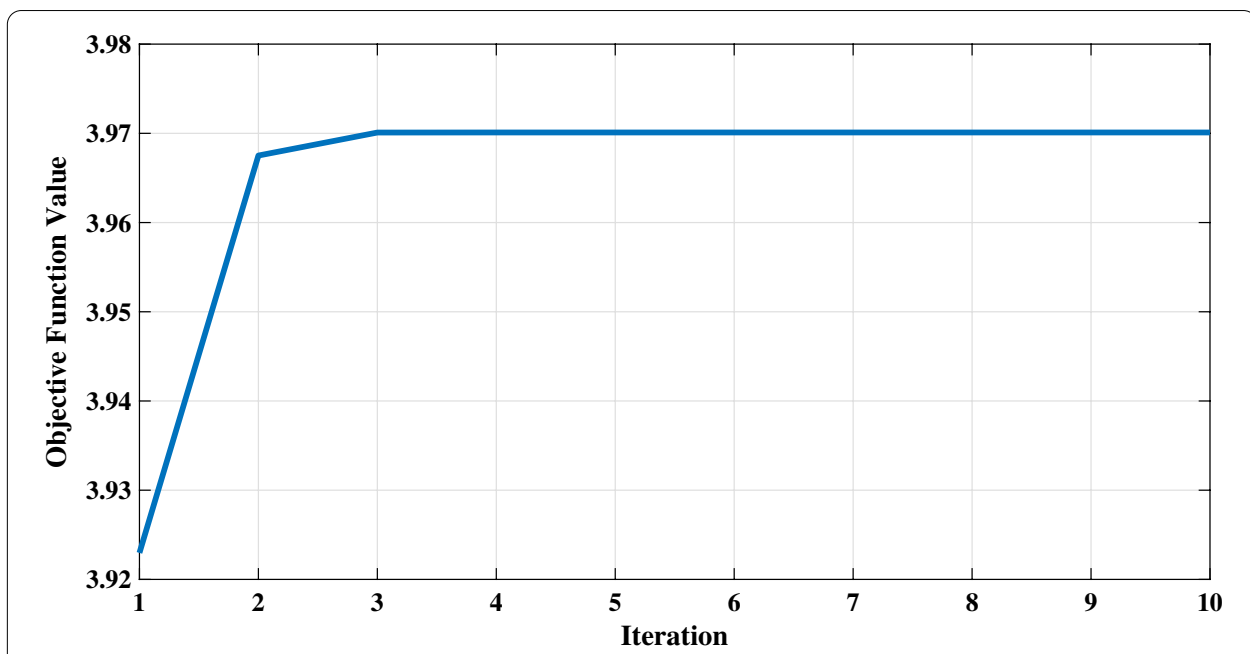

Fig. 5 Objective function value versus iteration number

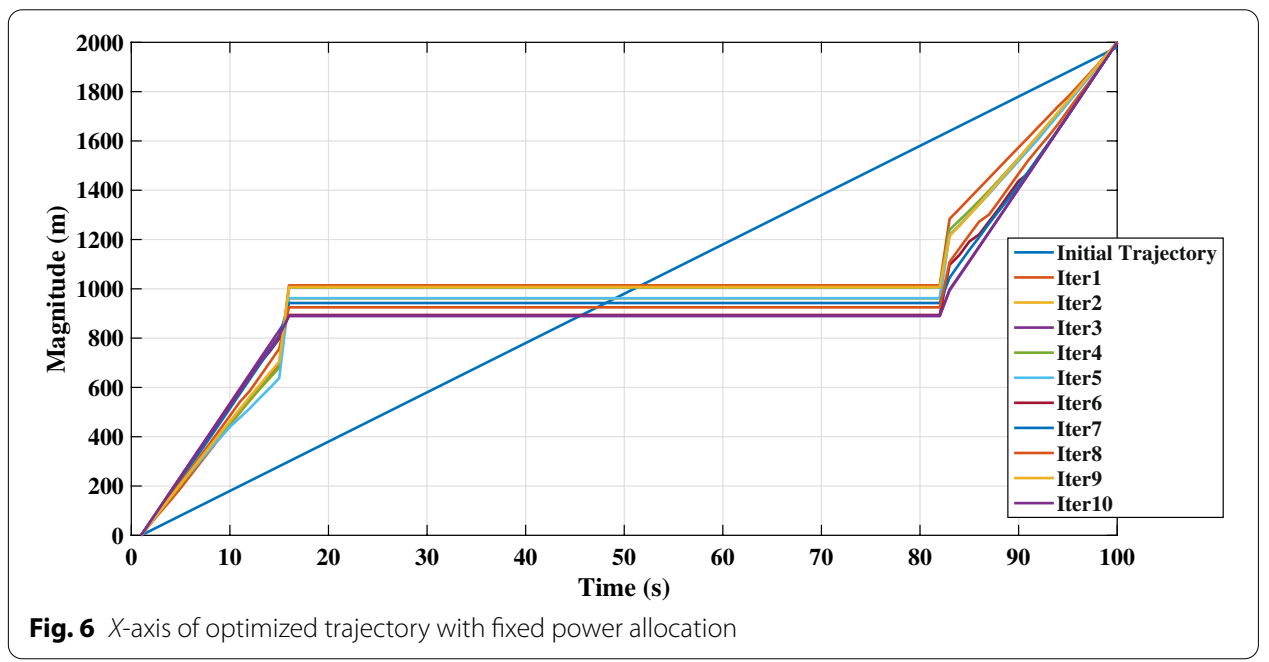

speed to a place near the middle of the trajectory and hovers there for the longest time because the maximum information rate can be achieved there. The convergence of the output can be seen obviously. Both $x$ - and $y$-axis of the UAV for the last iteration are plotted in Fig. 7. As we see, the optimal $y$-axis is equal to zero. The reason is that it is favorable that link distance reaches a minimum and lower power consumption we have.

In the following figures, the optimal information rate by the second scenario and the value of objective function for the optimization algorithm are plotted. As we know, in this scenario, the objective function is gained from Taylor expansion of information rate. The value of converged objective function $R_{l b, l+1}[n]$ in Fig. 8 is equal to 3.485 which is lower than its equivalent rate in Fig. 9. This results show that using Taylor expansion gives a lower bound of our desired function. In Fig. 9, The information rate increases while UAV flying from $(0,0,0)$ and gets fixed, as the location of UAV is fixed in Fig. 7 from 17 to 83 s. Figure 9 also indicates that when the UAV flies in the middle 


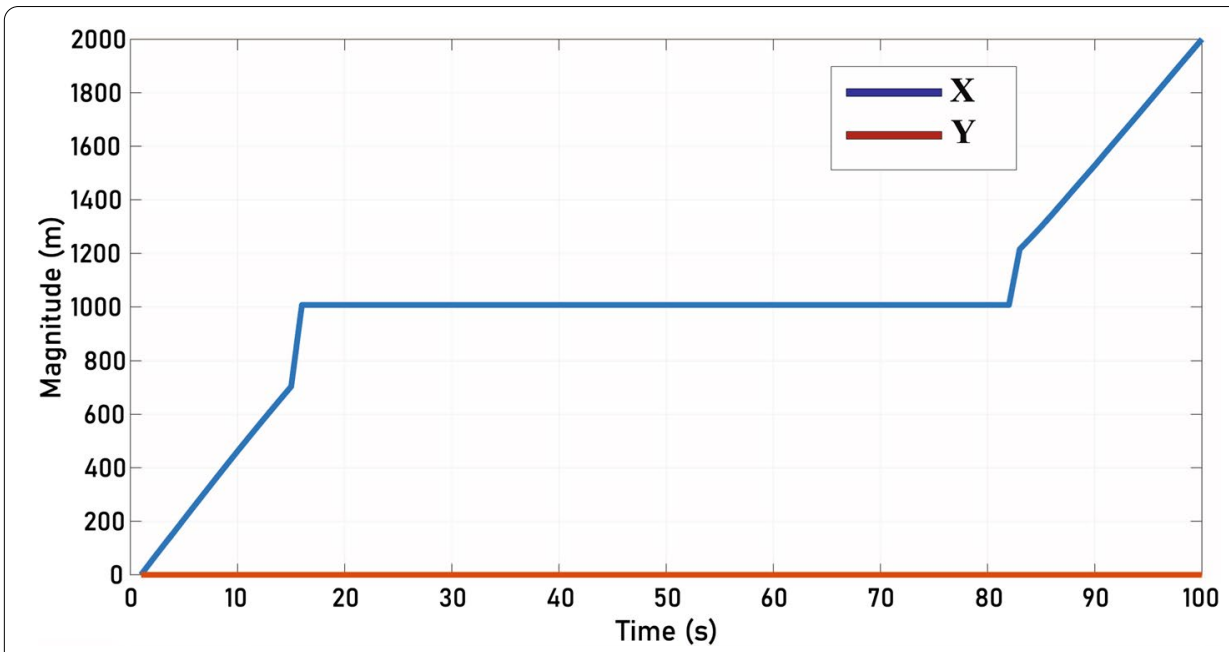

Fig. $7 X$-and $Y$-axis of optimized trajectory with fixed power allocation

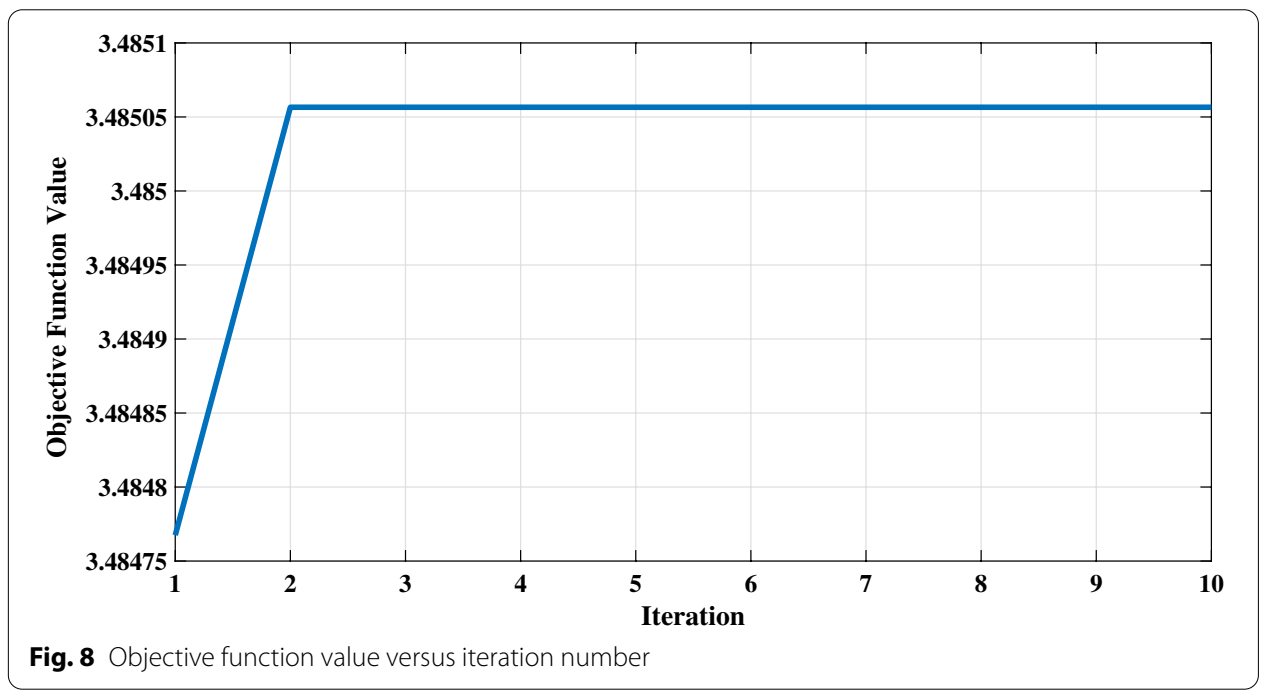

of the source and destination, the information rate has the maximum value. As you can see, the UAV is in the middle of the ground nodes longer than other times. This means that the UAV flies the most time where it has the highest rate.

In the above figures, only one parameter has been optimized. In this part, the results of joint power and trajectory optimization are presented. In Fig. 10, the optimized trajectory with $\mathrm{x}$ - and $\mathrm{y}$-axis is plotted. Like the previous part, the optimal $\mathrm{y}$ value is equal to zero due to minimizing link distance.

The UAV hovers for a long-time horizon from 17 to $83 \mathrm{~s}$ in the position of $900 \mathrm{~m}$. According to the expression for calculating information rate in (7), the information rate reaches maximum in this place with pre-determined $\bar{P}, \beta_{0}$ and $\sigma^{2}$. With this optimized trajectory, the power allocation scheme is shown in Fig. 11. 


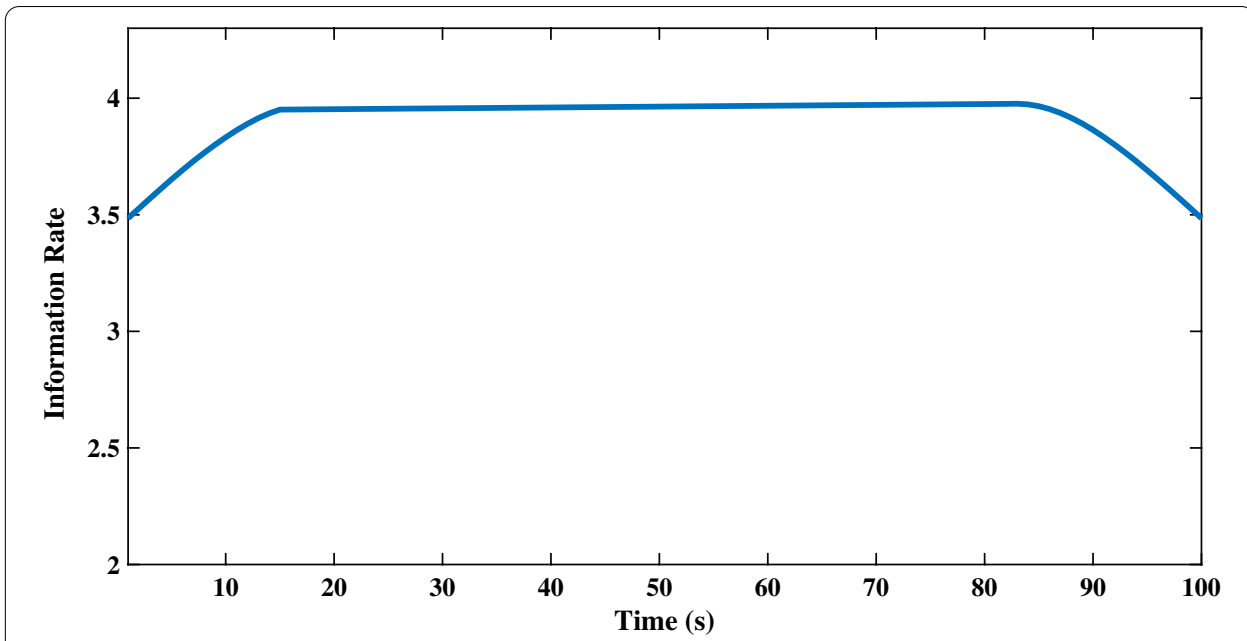

Fig. 9 Optimal information rate for the trajectory optimization with fixed power allocation

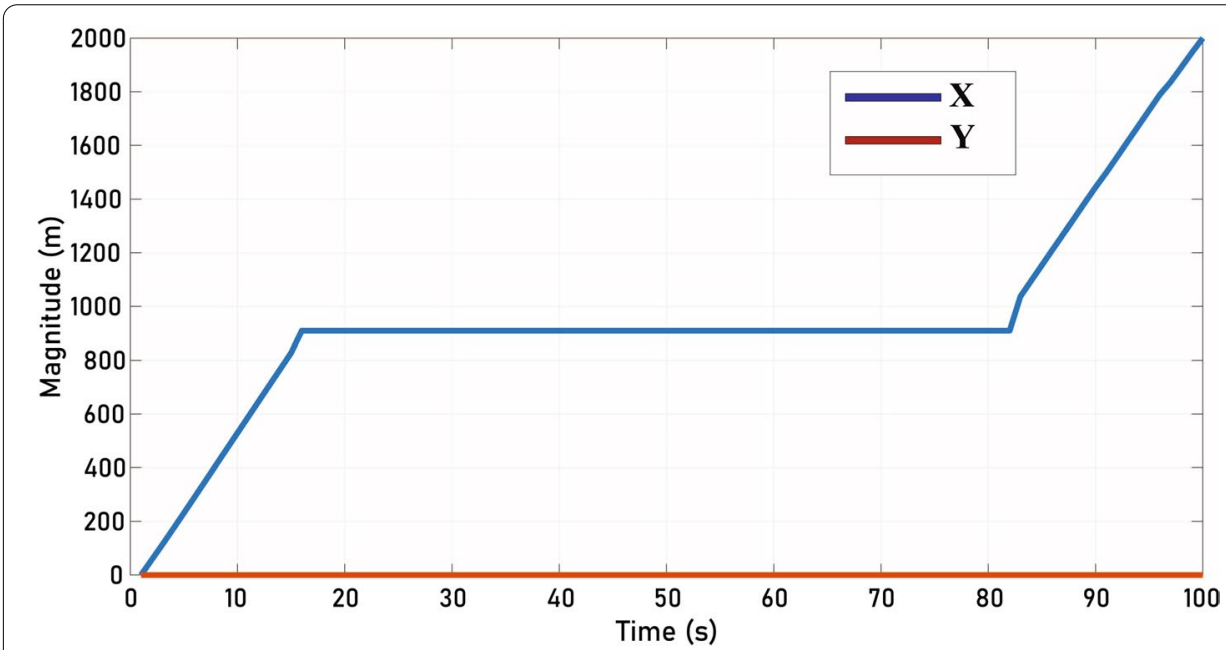

Fig. 10 Optimized trajectory by jointly optimizing power and trajectory

It is explainable that for the time that the UAV hovers in the middle of its trajectory, the power of source node A and UAV is almost equal. Before this time period, the UAV transmit with more power because it is close to $\mathrm{A}$ and its distance to $\mathrm{B}$ is more. From 83 to $100 \mathrm{~s}$, the transmission power of UAV decreases to $10 \mathrm{dBm}$, and the transmission power of A increases to $10 \mathrm{dBm}$ due to different link distances. In the last step, we compare the proposed algorithm with the case of fixed power and trajectory as a benchmark to evaluate the efficiency of proposed method. In the fixed power and trajectory case, the power of UAV and A are the same in the whole flying time, and UAV flies from (0, $0,100)$ to $(2000,0,100)$ with speed $20 \mathrm{~m} / \mathrm{s}$. As shown in Fig. 12, improvement of rate is achieved by jointly optimizing power and trajectory. 


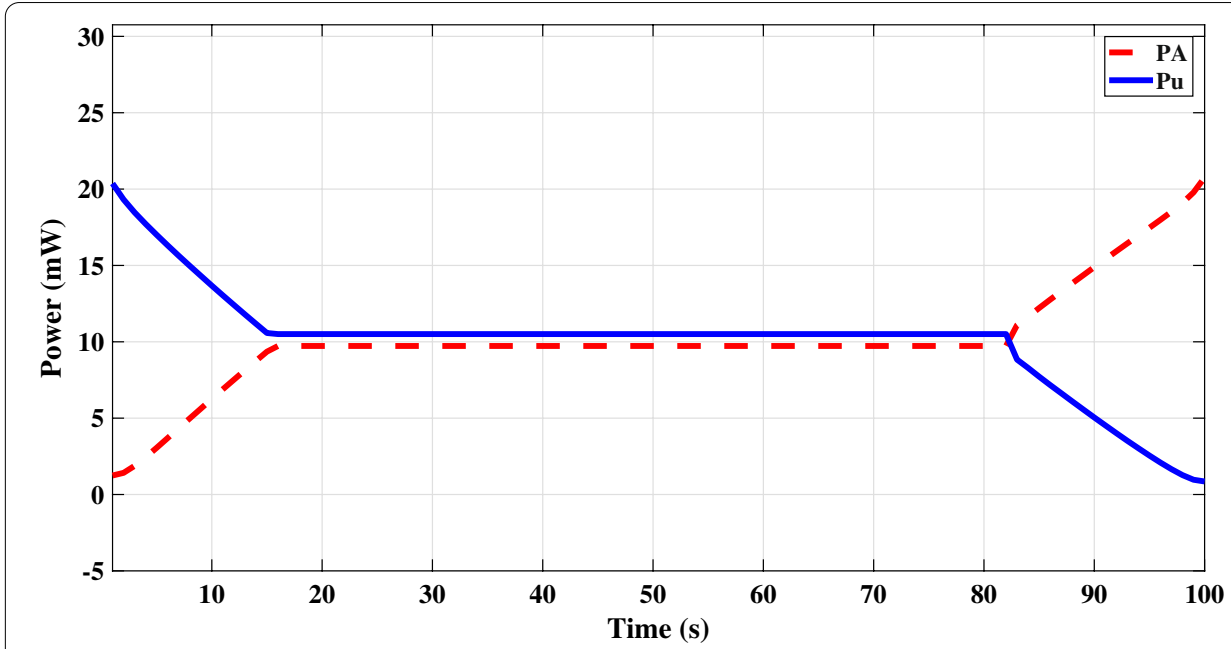

Fig. 11 Power allocation by jointly optimizing power and trajectory

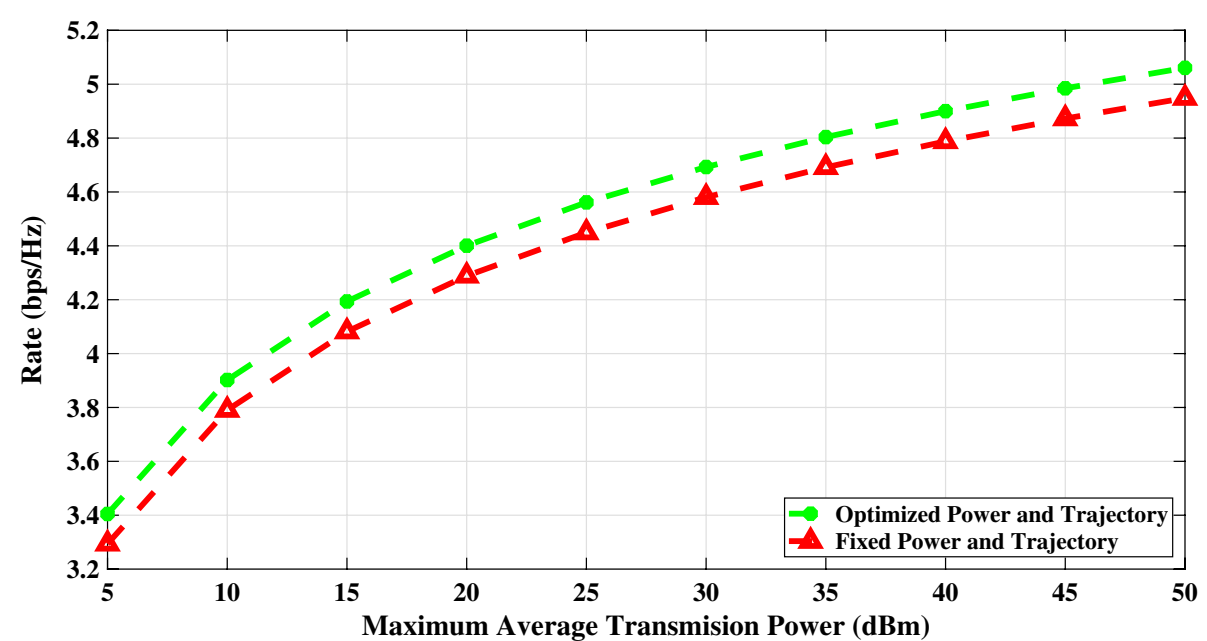

Fig. 12 Information rate with two power and trajectory scenario

\section{Conclusion}

In this paper, a UAV-based relaying system benefiting the UAV's mobility is studied. The minimum information rate of a considered wireless network is maximized via optimizing both the source/relay power allocation and relay trajectory. To this end, we propose two iterative algorithms for fixed trajectory and fixed power allocation scenarios and find the optimal solution for the lower bound of the maximum rate. According to the results of the proposed methods, an overall algorithm is derived which jointly optimizes the power allocations and UAV trajectory alternately. Simulation results demonstrate that a higher system rate can be achieved by considering mobile relay compared to static relay which is operational for future real wireless networks in temporary situations. For future work, we can intend interference scenarios and also NLOS channel caused by long buildings. 


\section{Abbreviations}

UAV: Unmanned aerial vehicle; 5G: Fifth-generation; loT: Internet of Things; 3GPP: Third-generation partnership project; LOS: Line-of-sight; GT: Ground terminals; WPT: Wireless power transfer; SWIPT: Simultaneous wireless information and power transfer; D2D: Device-to-device; SCO: Successive convex optimization; AF: Amplify-and-forward; DF: Decode-andforward; AWGN: Additive white Gaussian noise; SNR: Signal-to-noise ratio.

\section{Acknowledgments}

This work was supported by the Technology Grant of the Ministry of Science, Research and Technology of Iran and Information and Communications Technology Ministry of Iran (Grant No. 16-99-01-000197).

\section{Authors' contributions}

All authors contributed in designing the proposed schemes and also writing and reviewing the manuscript. The authors approved the final manuscript.

\section{Authors' information}

Seyed Masoud Mirrezaei received his B.Sc. degree in Communication Engineering from K. N. Toosi University of Technology in Sept. 2004. He received his M.Sc. and Ph.D. degrees in Electrical Engineering from Amirkabir University of Technology, Tehran, Iran, in 2007 and 2013, respectively. He has been as a PhD student and researcher in "Mobile and Wireless Networks Research Laboratory" in Amirkabir University of Technology under the supervision of Prof. Karim Faez. Also, he was a visiting research student in the Signal Design and Analysis Laboratory (SDAL) at Queen's University, Kingston, Canada, under the supervision of Prof. Shahram Yousefi from March 2011 to February 2012. He joined the Electrical Engineering Department of Shahrood University of Technology from 2013 up to now. His research interests lie in communications, cloud systems, big data, networks, information theory, signal processing, channel coding and network coding.

\section{Declarations}

Competing interests

The authors declare that they have no competing interests.

\section{Consent for publication}

All authors have agree and given their consent for submission of this paper to Euraship Journal of Wireless Communications and Networking.

Received: 6 July 2021 Accepted: 6 January 2022

Published online: 21 January 2022

\section{References}

1. Y. Zhao, R. Adve, T.J. Lim, Improving amplify-and-forward relay networks: optimal power allocation versus selection, in 2006 IEEE International Symposium on Information Theory (2006), pp. 1234-1238

2. X. Liu, Z. Li, N. Zhao, W. Meng, G. Gui, Y. Chen, F. Adachi, Transceiver design and multihop D2D for UAV IoT coverage in disasters. IEEE Internet Things J. 6(8), 1803-1815 (2018)

3. J. Xu, Y. Zeng, R. Zhang, UAV-enabled multiuser wireless power transfer: trajectory design and energy optimization, in 2017 23rd Asia-Pacific Conference on Communications (APCC) (2017), pp. 1-6

4. D. Yang, Q. Wu, Y. Zeng, R. Zhang, Energy tradeoff in ground-to-UAV communication via trajectory design. IEEE Trans. Veh. Technol. 67(7), 6721-6726 (2018)

5. R. Fan, J. Cui, S. Jin, K. Yang, J. An, Optimal node placement and resource allocation for UAV relaying network. IEEE Commun. Lett. 22(4), 808-811 (2018)

6. J. Lyu, Y. Zeng, R. Zhang, T.J. Lim, Placement optimization of UAV-mounted mobile base stations. IEEE Commun. Lett. 21(3), 604-607 (2016)

7. M. Mozaffari, W. Saad, M. Bennis, M. Debbah, Unmanned aerial vehicle with underlaid device-to-device communications: performance and tradeoffs. IEEE Trans. Wirel. Commun. 15(6), 3949-3963 (2016)

8. D.W. Matolak, R. Sun, Air-ground channel characterization for unmanned aircraft systems-part III: the suburban and near-urban environments. IEEE Trans. Veh. Technol. 66(8), 6607-6618 (2017)

9. 36.777, G.T.: Study on Enhanced LTE Support for Aerial Vehicles. https://www.3gpp.org/ftp/Specs/archive/36series/ $36.777 /$

10. Z. Yang, C. Pan, M. Shikh-Bahaei, W. Xu, M. Chen, M. Elkashlan, A. Nallanathan, Joint altitude, beamwidth, location, and bandwidth optimization for UAV-enabled communications. IEEE Commun. Lett. 22(8), 1716-1719 (2018)

11. Y. Chen, W. Feng, G. Zheng, Optimum placement of UAV as relays. IEEE Commun. Lett. 22(2), 248-251 (2017)

12. Q. Zeng, B. Zhong, J. Sun, C. Liu, M. Wang, Full-duplex relay aided device-to-device communication networks under the coverage of unmanned aerial vehicle base station, in 2018 24th Asia-Pacific Conference on Communications (APCC) (2018), pp. 196-200

13. Y. Li, H. Zhang, K. Long, C. Jiang, M. Guizani, Joint resource allocation and trajectory optimization with QoS in UAVbased NOMA wireless networks, in IEEE Transactions on Wireless Communications (2021)

14. Y. Zeng, R. Zhang, T.J. Lim, Throughput maximization for UAV-enabled mobile relaying systems. IEEE Trans. Commun. 64(12), 4983-4996 (2016)

15. X. Jiang, Z. Wu, Z. Yin, Z. Yang, Joint power and trajectory design for UAV-relayed wireless systems. IEEE Wirel. Commun. Lett. 8(3), 697-700 (2018)

16. Z. Sun, D. Yang, L. Xiao, L. Cuthbert, F. Wu, Y. Zhu, Joint energy and trajectory optimization for UAV-enabled relaying network with multi-pair users. IEEE Trans. Cogn. Commun. Netw. 78(3), 939-954 (2021) 
17. Y. Zeng, R. Zhang, Energy-efficient UAV communication with trajectory optimization. IEEE Trans. Wirel. Commun. 16(6), 3747-3760 (2017)

18. Y. Zeng, J. Xu, R. Zhang, Energy minimization for wireless communication with rotary-wing UAV. IEEE Trans. Wirel. Commun. 18(4), 2329-2345 (2019)

19. Q. Wu, Y. Zeng, R. Zhang, Joint trajectory and communication design for multi-UAV enabled wireless networks. IEEE Trans. Wirel. Commun. 17(3), 2109-2121 (2018)

20. J. Fan, M. Cui, G. Zhang, Y. Chen, Throughput improvement for multi-hop UAV relaying. IEEE Access 7, 147732 147742 (2019)

21. D. Cao, W. Yang, N. Li, Throughput enhancement of buffer-aided multi-hop UAV relaying wireless networks. AEU-Int. J. Electron. Commun. 131, 153596 (2021)

22. T. Zhang, J. Lei, Y. Liu, C. Feng, A. Nallanathan, Trajectory optimization for UAV emergency communication with limited user equipment energy: a safe-DQN approach. IEEE Trans. Green Commun. Netw. 5(3), 1236-1247 (2021)

23. X. Liu, Z. Li, N. Zhao, W. Meng, G. Gui, Y. Chen, F. Adachi, Transceiver design and multihop D2D for UAV IoT coverage in disasters. IEEE Internet Things J. 6(2), 1803-1815 (2018)

24. Z. Na, B. Mao, J. Shi, J. Wang, Z. Gao, M. Xiong, Joint trajectory and power optimization for UAV-relay-assisted internet of things in emergency. Phys. Commun. 41, 101100 (2020)

25. Q. Wang, Z. Chen, W. Mei, J. Fang, Improving physical layer security using UAV-enabled mobile relaying. IEEE Wirel. Commun. Lett. 6(3), 310-313 (2017)

26. M.T. Mamaghani, Y. Hong, Intelligent trajectory design for secure full-duplex MIMO-UAV relaying against active eavesdroppers: a model-free reinforcement learning approach. IEEE Access 9, 4447-4465 (2020)

27. M.-C. Mah, H.-S. Lim, A.W.-C. Tan, Secrecy improvement via joint optimization of UAV relay flight path and transmit power. Veh. Commun. 23, 100217 (2020)

28. X. Zhou, Q. Wu, S. Yan, F. Shu, J. Li, UAV-enabled secure communications: joint trajectory and transmit power optimization. IEEE Trans. Veh. Technol. 68(4), 4069-4073 (2019)

29. W. Lu, Y. Ding, Y. Gao, S. Hu, Y. Wu, N. Zhao, Y. Gong, Resource and trajectory optimization for secure communications in dual-UAV-mec systems. IEEE Trans. Ind. Inform. (2021)

30. S. BoydandL, Vandenberghe, convex optimization (Cambridge University Press, Cambridge, 2004)

\section{Publisher's Note}

Springer Nature remains neutral with regard to jurisdictional claims in published maps and institutional affiliations.

\section{Submit your manuscript to a SpringerOpen ${ }^{\circ}$ journal and benefit from:}

- Convenient online submission

- Rigorous peer review

- Open access: articles freely available online

- High visibility within the field

- Retaining the copyright to your article

Submit your next manuscript at $\gg$ springeropen.com 